\title{
Lipopolysaccharide Modification Accompanying Antigenic Conversion by Phage P27
}

\author{
By ALF A. LINDBERG \\ National Bacteriological Laboratory, S-105 21 Stockholm, Sweden \\ CARL G. HELLERQVIST \\ Department of Organic Chemistry, Stockholm University, Sweden \\ AND GENEVIÈVE BAGDIAN-MOTTA AND P. HELENA MÄKELÄ \\ Central Public Health Laboratory, Helsinki, Finland
}

(Received 8 February 1978)

\begin{abstract}
A structural study by methylation analysis was made of the lipopolysaccharides from Salmonella bredeney and strains of Salmonella typhimurium which had been lysogenized with phage P27 and thereby converted to have the new O-antigen factor 27 . The results were compared with previous studies on the non-converted, $27^{-}$, parental strains. The change from $27^{-}$to $27^{+}$was accompanied by a change in the linkage between the repeating units of the $O$ side-chain from galactose- $(1 \rightarrow 2)$-mannose to galactose- $(1 \rightarrow 6)$-mannose. In S. bredeney (P27), where the expression of antigen 27 is stable, $98 \%$ of the linkages were changed, whereas only 31 to $45 \%$ of the linkages were affected in two strains of $S$. typhimurium (P27). As 71 to $85 \%$ of the cells in the same batches of $S$. typhimurium bacteria were $27^{+}$it seems probable that in these strains many bacteria contained both types of linkages in their lipopolysaccharide.
\end{abstract}

\section{INTRODUCTION}

In Salmonella serogroups B and D some species isolated from natural sources have the antigen factor 27 and show the $O$-antigen formula (1), 4, (5), 12, 27 or (1), 9, 12, 27. Variants of the $27^{+}$species which lack factor 27 are found both in nature and in the laboratory. The presence of factor 27 has been shown to depend on a temperate phage, P27 (LeMinor et al., 1961), which converts both $27^{-}$variants of naturally $27^{+}$strains and strains normally $27^{-}$ into $27^{+}$(LeMinor, 1962). Factor 27 is a 'form variable' antigen factor (Kauffman \& Rhode, 1961): some strains exhibit this variation so that the same culture contains both $27^{+}$and $27^{-}$organisms, while other strains are stably $27^{+}$.

The chemical basis of factor 27 has been investigated in a Salmonella bredeney strain, by comparing a $27^{-}$variant and its $27^{+}$derivative obtained by lysogenization with $\mathrm{P} 27$ originally isolated from Salmonella schwarzengrund (Bagdian et al., 1966; Staub \& Bagdian, 1966). Factor 27, like other O-antigen factors, corresponds to a specific structural feature in the O-specific polysaccharide chain of the wall lipopolysaccharide (LPS) (Fig. 1). These chains are composed of oligosaccharide repeating units, which are polymerized into long chains attached to a carrier lipid before their final transfer to the LPS core (Osborn, 1969). The $27^{+}$and $27^{-}$strains differed in the linkage between repeating units so that factor 27 represents a galactose-( $1 \rightarrow 6)$-mannose linkage. In the $27^{-}$form the corresponding linkage was thought to be galactose-(1 $\rightarrow 4)$-mannose (Bagdian et al., 1966). Later, the structure of the O side-chain of Salmonella typhimurium, a naturally $27^{-}$strain of group B, was studied by more reliable analytical methods and the linkage between repeating units was shown to be 


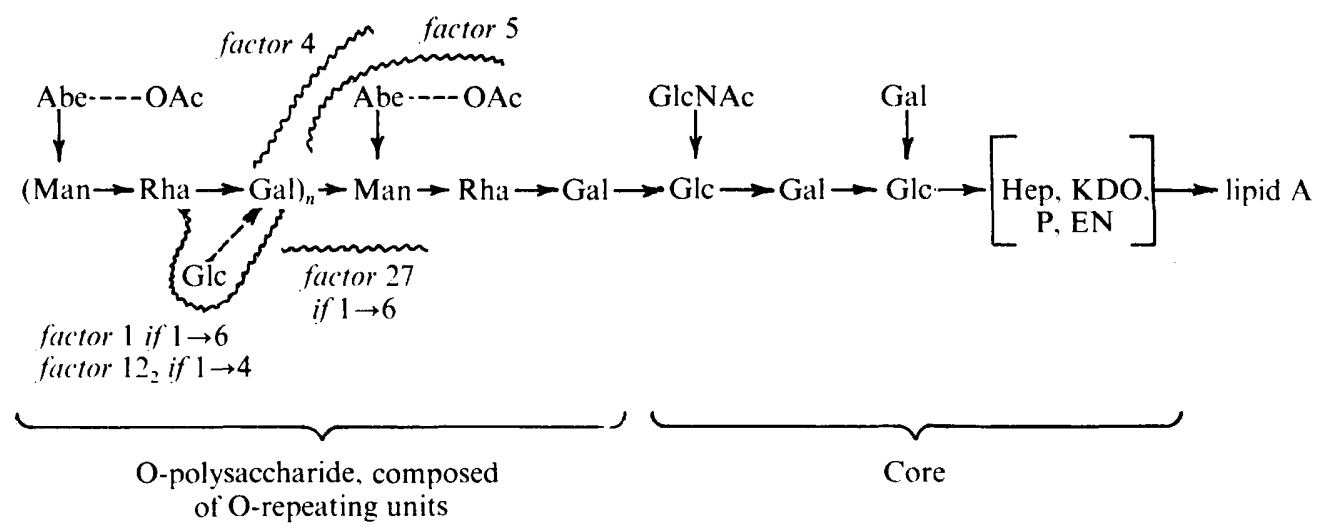

Fig. 1. Schematic structure of the LPS of $27^{+}$and $27^{-}$strains of Salmonella group B, O-antigens (1), $4,(5), 12,27^{+}$or $27^{-}$. Parentheses and dashed lines represent variable structures. Abe, abequose; EN, ethanolamine; Gal, D-galactose; Glc, D-glucose; GlcNAc, $N$-acetylglucosamine; Hep, heptose; KDO, 2-keto-3-deoxyoctonic acid; Man, D-mannose; OAc, $O$-acetyl; P, phosphate; Rha, L-rhamnose.

galactose-(1 $\rightarrow 2)$-mannose (Hellerqvist et al., 1968). This linkage was subsequently confirmed (Hellerqvist et al., 1969) in the 27- S. bredeney strain used by Bagdian et al. (1966). This discovery shed some suspicion on the original conclusion about the nature of factor 27 . We therefore decided to redetermine the O-antigenic polysaccharide structure in the $27^{+}$ $S$. bredeney strain.

It also appeared pertinent to examine the LPS structure in another $27^{+}$-converted Salmonella strain. We chose $S$. typhimurium, which is naturally $27^{-}$. In this strain, the factor 27 is variable when it is converted by $\mathrm{P} 27$, whereas it is stable in the $27^{+} S$. bredeney strain (Bagdian et al., 1966; Staub \& Bagdian, 1966; Nurminen et al., 1971). We could then compare the percentage of $27^{+}$-specific linkages with the percentage of detectably $27^{+}$ bacteria in the population of the two organisms and thus obtain a measure of the completeness of the conversion.

\section{METHODS}

Phages and phage methods. Phage P27, isolated from S. schwarzengrund (LeMinor, 1962), was propagated on Salmonella paratyphi в (Bagdian \& Mäkelä,1971). When used to infect S. typhimurium it proved to contain two separate phages, only one of which effected conversion to $27^{+}$. Therefore a $27^{+}$-converted lysogenic derivative of S. typhimurium (sL696, see below) was used as the source of re-isolated phage P27. This phage, when grown on the $S$. paratyphi B strain, lysogenized $S$. typhimurium efficiently so that most lysogenic colonies were $27^{+}$.

The attachment rate constant of phages P22 and P27 to parent strains as well as to lysogenized bacteria was estimated as described previously by determining the number of unadsorbed phages in a chloroformtreated fraction (Lindberg \& Holme, 1969). Data reported are the mean value of two experiments with each bacterial strain.

Bacteriological methods. Salmonella bredeney SH363, a derivative of the $S$. bredeney line B15-60 used in previous studies (LeMinor et al., 1961; Bagdian et al., 1966; Staub \& Bagdian, 1966; Hellerqvist et al., 1969; Bagdian \& Mäkelä, 1971) was converted (Bagdian \& Mäkelä, 1971) by lysogenization with phage P27 to O-antigen 27 specificity to yield strain SH363(P27). Derivatives of the sD14 subline (Spicer \& Datta, 1959) of S. typhimurium LT2 were likewise converted by lysogenization with P27 to yield strains SH2183(P22,P27), derived from SH2183 and also lysogenic for P22 (Nurminen et al., 1971), and sH5110, derived from sL696 (Spicer \& Datta, 1959) and not lysogenic for P22 (Table 1).

The O-antigens of the bacteria were tested by slide agglutination (Kauffmann, 1966) in appropriately diluted antisera and in $4 \%(\mathrm{w} / \mathrm{v})$ saline to exclude rough forms. The individual antisera for $\mathrm{O}$-antigen factors $1,4,5,12$ and 27 were prepared in rabbits and absorbed to specificity by the methods of Kauffmann (1966).

The bacteria were grown at $37^{\circ} \mathrm{C}$ in submerged culture in a complex medium to late exponential phase 
Table 1. Strains of Salmonella bredeney (line B15-60) and S. typhimurium (line LT2, subline SD14) used in this study

\begin{tabular}{|c|c|c|c|c|c|c|c|c|}
\hline \multirow[b]{2}{*}{ Organism } & \multirow[b]{2}{*}{ Strain } & \multicolumn{2}{|c|}{ Prophages } & \multicolumn{5}{|c|}{ Agglutination* in factor antisera } \\
\hline & & $\mathbf{P} 22$ & $\mathbf{P} 27$ & 1 & 4 & 5 & 12 & 27 \\
\hline S. bredeney & SH363 & - & - & + & + & - & + & - \\
\hline S. bredeney & SH363(P27) & - & + & + & + & - & + & + \\
\hline S. typhimurium & SH2183(P22) & + & - & \pm & + & + & + & - \\
\hline S. typhimurium & $\mathrm{sH} 2183(\mathrm{P} 22, \mathrm{P} 27)$ & + & + & $\overline{-}$ & + & + & + & + \\
\hline S. typhimurium & SH5110(P27) & - & + & - & + & + & + & + \\
\hline
\end{tabular}

(Hellerqvist et al., 1968). Samples were taken before harvesting and spread on nutrient agar plates (Difco). After overnight incubation, isolated colonies were tested by slide agglutination against antisera to $\mathrm{O}$ factors 4 , 5 and 27 and against $4 \%$ saline. The supernatant fluid obtained after harvesting the submerged culture was analysed for the content of either phage P27 or phages P27 and P22 using appropriate indicator strains.

Extraction of lipopolysaccharide and separation of polysaccharide. LPS was extracted by hot phenol/water (Westphal et al., 1952) from formaldehyde-killed bacteria (Lindberg \& Holme, 1972) and purified by repeated cycles of ultracentrifugation at $105000 \mathrm{~g}$ for $4 \mathrm{~h}$ until virtually free from specific absorption at $260 \mathrm{~nm}$. The LPS fractions were then hydrolysed in aqueous acetic acid, pH $3 \cdot 2$, at $100{ }^{\circ} \mathrm{C}$ for $60 \mathrm{~min}$ to split the acidlabile 3-deoxyoctulosonic linkages in the basal core (Dröge et al., 1968). This resulted in a polysaccharide fraction free from lipid A. The polysaccharide was fractionated on a Sephadex G-50 column with 0.05 Mpyridine/acetate buffer, $\mathrm{pH} 5 \cdot 5$, as eluant. The fractions were analysed for carbohydrate content using the phenol-sulphuric acid method (Whistler \& Wolfrom, 1962). The peak fractions were pooled and subjected to chemical analyses.

Analysis of monosaccharides. The pooled fractionated polysaccharides were hydrolysed to give a mixture of monosaccharides which were reduced with an excess of sodium borohydride at room temperature for $16 \mathrm{~h}$. Excess borohydride was destroyed by addition of Dowex $50\left(\mathrm{H}^{+}\right)$and boric acid was removed as methyl borate by several distillations with methanol under reduced pressure. The syrups were dried by distillation under reduced pressure with benzene, and then fully acetylated with acetic anhydride/pyridine $(1: 1, \mathrm{v} / \mathrm{v})$ at $100{ }^{\circ} \mathrm{C}$ for $1 \mathrm{~h}$. Excess acetic anhydride was removed by co-distillation with toluene. The resulting mixture of fully acetylated alditol acetates was dissolved in chloroform and analysed by gas-liquid chromatography (Sawardeker et al., 1965). The different sugars were identified by their retention times ( $\mathrm{T}$ values) and mass spectra (Golovkina et al., 1966).

Analysis of polysaccharide structure. The fractionated polysaccharides were methylated, and the methylated sugars were converted to alditol acetates and analysed by gas-liquid chromatography (Björndal et al., $1967 a, b)$. The components were identified by mass spectrometry and corresponding retention times; $\mathrm{T}$ values are given relative to 1,5 -di- $O$-acetyl-2,3,4,6-tetra- $O$-methyl-D-glucitol.

Presence of $O$-acetyl groups. This was investigated by infrared spectrometry. The spectral evidence was corroborated by looking for methyl acetate by gas-liquid chromatography/mass spectrometry after treating the LPS preparations with $4 \%(\mathrm{v} / \mathrm{v})$ methanolic $\mathrm{HCl}(2 \mathrm{mg} \mathrm{LPS} \mathrm{in} 0.5 \mathrm{ml})$ for $1 \mathrm{~h}$ at $100^{\circ} \mathrm{C}$.

Alkali-labile groups. The presence of these groups was investigated in the fractionated polysaccharide according to the method of DeBelder \& Norrman (1968). The fully acetylated polysaccharide was then methylated and analysed as described earlier (Hellerqvist et al., 1968).

\section{RESULTS}

\section{Serology and stability of the strains}

The $S$. bredeney $27^{-}$strain was completely stable; all its colonies agglutinated in anti-1, anti-4 and anti-12 factor sera (Table 1). The converted $S$. bredeney (P27) was likewise stable; in the batch from which the LPS was extracted 50 out of 50 colonies gave a strong agglutination in anti-27 serum as well as in anti-1, anti-4 and anti-12 factor sera.

Salmonella typhimurium strains, when converted by P27, showed rapid form variation. The batch culture for preparing the LPS was always started from a fresh colony which gave a strong agglutination reaction in anti-27 serum. Nevertheless, a large fraction of the colonies grown from the final batch were $27^{-}: 23 \%$ in SH2183(P22,P27), $15 \%$ in sH5110 (Table 1). In 
Table 2. Monosaccharide content (molar proportions of detected sugars) of the five LPS preparations studied

\begin{tabular}{|c|c|c|c|c|c|c|}
\hline \multirow[b]{2}{*}{ Component } & \multirow[b]{2}{*}{ Derived from* } & \multicolumn{2}{|c|}{ S. bredeney } & \multicolumn{3}{|c|}{ S. typhimurium } \\
\hline & & $27^{-}$ & $27^{+}$ & $27^{-}$ & $27^{+} \dagger$ & $27^{+} \ddagger$ \\
\hline Abequose & O-PS & 20 & 18 & 19 & 9 & 12 \\
\hline L-Rhamnose & O-PS & 22 & 18 & 20 & 14 & 18 \\
\hline D-Mannose & O-PS & 23 & 18 & 19 & 14 & 18 \\
\hline D-Galactose & O-PS and core & 25 & 25 & 30 & 18 & 21 \\
\hline D-Glucose & O-PS and core & 10 & 14 & 12 & 12 & 6 \\
\hline Heptose & core & 3 & 6 & ND & 5 & ND \\
\hline
\end{tabular}

ND, Not detected.

* O-PS, O-polysaccharide; core, core polysaccharide; see Fig. 1.

$\uparrow$ Strain sH2183(P22,P27). $¥$ Strain sH5110(P27).

strain SH2183 the phage P22 determined the production of antigenic factor 1, which is based on glucose $(1 \rightarrow 6)-\alpha$-linked to galactose in the O-repeating unit (Stocker et al., 1960). This factor is also form variable.

\section{Lipopolysaccharide analysis}

The overall compositions of the LPS of the strains examined are given in Table 2 and are as expected. Abequose, mannose and rhamnose derive exclusively from the O-repeating units and should occur in equal amounts when the acid-lability of abequose is accounted for (Hellerqvist et al., 1968). When this was not done, however, lower values for abequose were observed (Table 2). Galactose occurs both as an integral component of the repeating units and in the core, and should therefore be found in slightly higher amounts than the other O-specific sugars. Glucose is an integral component of the core (where it occurs in equimolar amounts with galactose) and is also present as a variable branch substituent of the O-polysaccharide. As expected, it was found in variable amounts and, on the basis of the methylation analyses (see below), corresponded to between 0 and $30 \%$ substitution of the galactose units of the O-polysaccharide.

The antigenic factor 5 is absent in $S$. bredeney but present in $S$. typhimurium where it is based on an alkali-labile group at $O-2$ in abequose (Kotelko et al., 1961). We found evidence (see Methods) of $O$-acetyl substituents in all three strains of $S$. typhimurium but not in the two $S$. bredeney strains. Neither could we find any alkali-labile groups (DeBelder \& Norrman, 1968 ) in the $S$. bredeney strains.

The structure of the O-polysaccharides was studied by methylation analysis. The molar percentages of the different methyl ethers of the $27^{+}$LPS are given in Table 3, together with those obtained from the $27^{-}$LPS. From the mass spectra and T values, most peaks could be unambigously identified. Peak B revealed the presence of both a 6-deoxy-2,3-di- $O$-methylhexose and a 2,3,4,6-tetra- $O$-methylhexose. These components were fully separated and identified on a OS138 (polyphenylether) column as 2,3-di-O-methyl-L-rhamnose and 2,3,4,6-tetra- $O$-methyl-D-glucose.

The major difference in the analyses of $27^{-}$and $27^{+}$LPS was the presence of peak $M$ in $27^{+}$LPS. The retention time and mass spectra of peak $M$ identified it as a derivative of 2,4-di-O-methyl-D-mannose. In the $S$. bredeney SH363(P27) LPS, this peak corresponded to $90 \%$ of the total mannose, the remainder being divided between 4,6-di- $O$-methyl-D-mannose (peak $\mathrm{H}$ ) and 2,4,6-tri- $O$-methyl-D-mannose (peak E). In analysis of the 27- LPS, no 2,4-di- $O$-methyl-D-mannose was found; the mannose was here found as 4,6-di- $O$-methylD-mannose and 2,4,6-tri- $O$-methyl-D-mannose. It can be assumed that in the $27^{+} \mathrm{LPS}$ the 2,4-di- $O$-methyl-D-mannose is derived from mannose in the phage 27-converted galactose$(1 \rightarrow 6)$-mannose linkage between the repeating units. The 4,6-di- $O$-methyl-D-mannose represents the non-converted $27^{-}$galactose-( $\left.1 \rightarrow 2\right)$-mannose linkage, and 2,4,6-tri- $O$-methyl- 


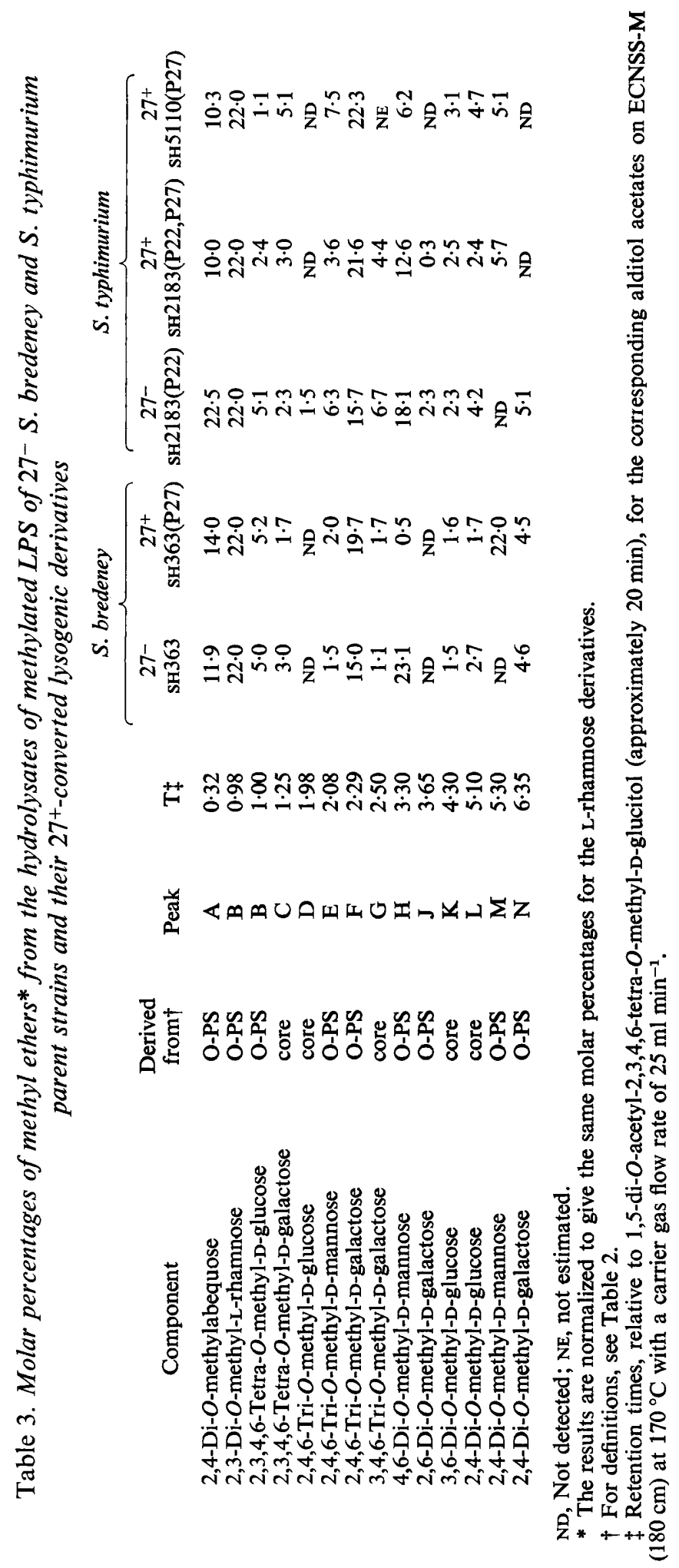


Table 4. Adsorption rate constants of the $\mathrm{P} 22$ and $\mathrm{P} 27$ bacteriophages to parent and converted $S$. bredeney and $S$. typhimurium strains

$\begin{array}{lcc}\text { Strain } & \overbrace{}^{\text {P22 }} & \begin{array}{l}10^{11} \times \text { Adsorption rate } \\ \text { constant }\left(\mathrm{ml} \mathrm{min}^{-1}\right)\end{array} \\ \text { S. bredeney } \text { SH363 } & 402 & 260 \\ \text { S. bredeney SH363(P27) } & <5 & <5 \\ \text { S. typhimurium LT2 } & 297 & \text { ND } \\ \text { S. typhimurium SH2183(P22) } & 41 & 312 \\ \text { S. typhimurium } \text { SH2183(P22,P27) } & 52 & 228\end{array}$

ND, Not determined.

D-mannose the methyl ether which originates from the mannose residue which terminates each $O$ side-chain (see Fig. 1). In the $27^{+}$-converted $S$. typhimurium strains, we likewise found 2,4-di- $O$-methyl-D-mannose which was absent from the corresponding $27^{-}$LPS.

The relative amount of 2,4,6-tri- $O$-methyl-D-mannose compared with total mannose allowed us to calculate the average number of repeating units per O-polysaccharide chain. These are 16 and 12, respectively, for the $27^{-}$and $27^{+} S$. bredeney strains, 4 for the $27^{-}$ S. typhimurium, and 6 and 3, respectively, for its two $27^{+}$derivatives. The distribution of 4,6-di- $O$-methyl-D-mannose and 2,4-di- $O$-methyl-D-mannose shows that in the $27^{+} S$. bredeney the conversion is almost complete: $98 \%$ of the linkages between repeating units are of the $27^{+}$type [galactose-(1-6)-mannose]. The situation is quite different in the two $27+$ S. typhimurium strains where only 31 to $45 \%$ of the linkages are of this type.

Some other differences were seen between the LPS preparations. 2,4,6-Tri- $O$-methyl-Dglucose (peak D) was found only in the $27-S$. typhimurium strain and may derive from an incomplete core (Hellerqvist \& Lindberg, 1971). 2,6-Di- $O$-methyl-D-galactose (the minor peak $\mathbf{J}$ ) originates from the galactose residue in the O-repeating unit which carries D-glucose linked to C-4, determining O-12 2 specificity (Tinelli \& Staub, 1960). This peak was not found in the two $S$. bredeney strains, consistent with the reported absence of antigen $12_{2}$ in $S$. bredeney. In the $27^{-} S$. typhimurium it represented $10 \%$ of the repeating unit galactosyl residues, much less in the $27^{+}$strain SH2183(P22,P27), and was absent from the other $27^{+}$ derivative sH5110(P27). Finally peak $N$ was present in all the LPS samples except the two from the P27-converted $S$. typhimurium strains. It corresponds to $\mathrm{O}$-antigen 1 , which is thus present on the average on every fourth or fifth repeating unit; antigen 1 was detected by agglutination in the three strains whose LPS yielded peak D.

\section{Effect of phage P27 conversion on phage attachment}

The smooth-specific P22 phage lyses only Salmonella strains that have O-antigen 12, i.e. serogroups A, B and D (Zinder \& Lederberg, 1952). Common to these bacteria is a galactose$(1 \rightarrow 2)-\alpha$-mannose linkage between the repeating units (Hellerqvist et al., 1971 a,b,c). Furthermore, the O-chain must consist of more than one repeating unit (Naide et al., 1965). The entire P22 receptor is found in the extracted LPS (Lindberg \& Hellerqvist, 1971). Phage P27 also attacks only smooth strains of serogroups A, B and D which have the O-antigen 12. However, its attachment site or receptor has not been studied. The change of linkage between the repeating units in the $27^{+}$-converted $S$. bredeney strain abolished the attachment of both phages P22 and P27 (Table 4). The $S$. typhimurium SH2183(P22) strain, lysogenic for P22, adsorbs this phage with a much reduced efficiency. The P27 conversion did not affect the P22 attachment rate in a measurable way while the P27 attachment rate was slightly decreased. This result probably reflects the fact that only every third linkage had been converted by phage P27. 


\section{DISCUSSION}

The earlier observation that the linkage galactose- $(1 \rightarrow 6)$-mannose corresponds to $27^{+}$ appears to be correct. The physiological repeating unit in biosynthesis is abequose $\rightarrow$ mannose $\rightarrow$ rhamnose $\rightarrow$ galactose (Nikaido, 1965; Nikaido \& Nikaido, 1965), and so the action of P27 concerns the linkage between repeating units, which is changed from $(1 \rightarrow 2)$ to $(1 \rightarrow 6)$. The conversion was the same in both the naturally occurring $27^{+} S$. bredeney and in $27^{+} S$. typhimurium which is never found in nature. The P27-converted $S$. bredeney was completely stable. Calculation of the number of converted linkages (2,4-di- $O$-methyl-D-mannose/ 4,6-di- $O$-methyl-D-mannose) showed that only $2 \%$ of the linkages were galactose-( $1 \rightarrow 2)$ mannose instead of galactose-( $1 \rightarrow 6)$-mannose.

In contrast, P27 lysogenization of $S$. typhimurium never resulted in stable $27^{+}$strains. In the two $27+S$. typhimurium derivatives studied here, only 71 to $85 \%$ of the colonies were $27^{+}$although the cultures had been grown from a single $27^{+}$colony. This represents form variation of the antigen 27 ; the P27 phage was still found in the supernatants. In the corresponding LPS preparations the number of converted galactose-(1 $\rightarrow 6)$-mannose linkages was 31 to $45 \%$, respectively, while the remaining 69 to $55 \%$ were still of the parental galactose- $(1 \rightarrow 2)$-mannose type. Therefore many $27^{+}$bacteria probably contained LPS with both types of linkages in their O-chains.

Salmonella bredeney has antigen 1 which is not form variable. In the O-chains of both $27^{+}$ and $27^{-}$strains every fourth or fifth galactose residue was substituted at C- 6 by glucose which determines O-antigen 1 specificity (Stocker et al., 1960). Thus phage P27 does not interfere with the glucosylation of the repeating unit suggesting that this type of glucosylation can take place irrespective of the neighbouring galactose-mannose linkage and certainly when it is either $(1 \rightarrow 2)$ or $(1 \rightarrow 6)$.

On the other hand, the antigen 1 in $S$. typhimurium is determined by phage P22 (Stocker et al., 1960) and is form variable. The $27^{-}$strain was lysogenic for P22 and therefore inherently capable of antigen 1 synthesis; correspondingly, in its LPS every fourth galactose of the O-repeating units was substituted by glucose at C- 6 .

The change of the galactose-(1 $\rightarrow 2)$-mannose linkage to galactose-(1 $1 \rightarrow 6)$-mannose between the repeating units in the $O$-polysaccharide chains of the $27^{+}$bacteria alters the stereochemistry and probably the biological properties of the LPS. A change is observed in its antigenic properties as appearance of a new antigen factor 27 . In strains where the conversion to the new $(1 \rightarrow 6)$ linkage is nearly complete, as in $S$. bredeney, it is very probable that some antigenic features associated with the $(1 \rightarrow 2)$ linkage are lost. This has, however, never been specifically tested.

The altered LPS structure also alters the reactivity of the LPS as phage receptor. Both P22 and $\mathrm{P} 27$ attach to the O-chains of the LPS in serogroups A, B and D, a reaction that can be measured as inactivation of free phage (Lindberg \& Hellerqvist, 1971). Thus the conversion of the linkage between the repeating units in $S$. bredeney resulted in abolition of the attachment of both P22 and P27. In $27^{+} S$. typhimurium, where only every third linkage was converted, the effect was less dramatic and both P22 and P27 were found to attach. Only the adsorption of P27 was reduced compared with the $27^{-}$parent strain; the adsorption of P22 was low in both strains due to P22 lysogeny.

The skilled technical assistance of Jana Cederstrand, Gunnel Engström and Anita Eriksson is gratefully acknowledged. This work was supported by grants from the Swedish Medical Research Council (40X-656) and the Finnish Medical Research Council. G. B-M. was a fellow of INSERM. 


\section{REFERENCES}

Bagdian, G. \& MäKelä, P. H. (1971). Antigenic conversion by phage P27. I. Mapping of the prophage attachment site on the Salmonella chromosome. Virology 43, 403-411.

BAgdian, G., LÜDERITZ, O. \& STAUB, A. M. (1966). Immunochemical studies on Salmonella. XI. Chemical modification correlated with conversion of group B Salmonella by bacteriophage 27. Annals of the New York Academy of Sciences 133, 405-424.

BJörndal, H., LindBERG, B. \& SVEnSSON, S. (1967a). Gas-liquid chromatography of partially methylated alditols as their acetates. Acta chemica scandinavica 21, 1801-1804.

BJöRNDAL, H., LINDBERG, B. \& SvenSSON, S. (1967b). Mass spectrometry of partially methylated alditol acetates. Carbohydrate Research 5, 433-440.

DeBelder, A. N. \& Norrman, B. (1968). The distribution of substituents in partially acetylated dextran. Carbohydrate Research 8, 1-6.

Dröge, W., RuschmanN, E., Lüderitz, O. \& WestPhal, O. (1968). Biochemical studies on lipopolysaccharides of Salmonella $\mathrm{R}$ mutants. 4. Phosphate groups linked to heptose units and their absence in some $\mathrm{R}$ lipopolysaccharides. European Journal of Biochemistry 4, 134-138.

Golovkina, L. S., Chizov, O. S. \& Wulfson, N. S. (1966). Mass spectrometer investigation of carbohydrates. Communication 9. Acetates of polyols. Bulletin of the Academy of Sciences of the USSR. Division of Chemical Science 11, 1853-1863.

HellerQvist, C. G. \& LindBerG, A. A. (1971). Structural studies of the common-core polysaccharide of the cell-wall lipopolysaccharide from Salmonella typhimurium. Carbohydrate Research 16, 39-48.

Hellerqvist, C. G., Lindberg, B., Svensson, S., Holme, T. \& LindberG, A. A. (1968). Structural studies on the O-specific side-chains of the cellwall lipopolysaccharide of Salmonella typhimurium 395Ms. Carbohydrate Research 8, 43-55.

HellerQvist, C. G., Larm, O., LindBerG, B., Holme, T. \& LindberG, A. A. (1969). Structural studies on the O-specific side-chains of the cellwall lipopolysaccharide from Salmonella bredeney. Acta chemica scandinavica 23, 2217-2222.

Hellerqvist, C. G., LARM, O., LindberG, B. \& LINDBERG, A. A. (1971 a). Structure of an oligosaccharide obtained on degradation of the lipopolysaccharide from Salmonella typhimurium LT2. Acta chemica scandinavica 25, 744-745.

Hellerovist, C. G., LindberG, B., SAmuelsson, K. \& LINDBERG, A. A. (1971 b). Structural studies on the $O$ specific side-chains of the cell-wall lipopolysaccharide from Salmonella paratyphi A var. durazzo. Acta chemica scandinavica 25, 955-961.

Hellerqvist, C. G., Hoffman, J., LindberG, B., PilotTi, A. \& Lindberg A. A. (1971c). Anomeric nature of the D-mannose residues in the Salmonella typhi and S. strasbourg lipopolysaccharides. Acta chemica scandinavica 25, 1512-1513.

Kauffman, F. (1966). Die Bakteriologie der Salmonella Species. Copenhagen: Munksgaard.

KaUfFMANN, F. \& Rhode, R. (1961). Neue Befunden beim O-Formenwechsel der Salmonella Species. Acta pathologica et microbiologica scandinavica 52, 211-216.

Kotelko, K., Staub, A. M. \& Tinelli, R. (1961). Étude immunochimique des Salmonella. VIII. Rôle des groupements O-acétyls dans la spécificité du facteur 0:5. Annales de l'Institut Pasteur 100, 618-637.

LEMINOR, L. (1962). Conversion par lysogénisation de quelques sérotypes de Salmonella des groupes $\mathrm{A}, \mathrm{B}$ et $\mathrm{D}$ normalement dépourves du facteur O:27 en cultures 27 positives. Annales de l'Institut Pasteur 103, 684-706.

LeMinor, L., LeMinor, S. \& Nicolle, P. (1961). Conversion de cultures de Salmonella schwarzengrund et Salmonella bredeney en cultures 27 positives par la lysogénisation. Annales de l'Institut Pasteur 101, 571-589.

LindBerg, A. A. \& Hellerqvist, C. G. (1971). Bacteriophage attachment sites, serological specificity, and chemical composition of the lipopolysaccharides of semirough and rough mutants of Salmonella typhimurium. Journal of Bacteriology 105, 57-64.

LiNDBERG, A. A. \& Holme, T. (1969). Influence of O side-chains on the attachment of the Felix O-1 bacteriophage to Salmonella bacteria. Journal of Bacteriology 99, 513-519.

LindBerg, A. A. \& Holme, T. (1972). Evaluation of some extraction methods for the preparation of bacterial lipopolysaccharides for structural analysis. Acta pathologica et microbiologica scandinavica B 80, 751-759.

NAIDE, Y., Nikaido, H., Mäkelä, P. H., WilkINSON, R. G. \& Stocker, B. A. D. (1965). Semirough strains of Salmonella. Proceedings of the National Academy of Sciences of the United States of America 53, 147-153.

NikaIDo, H. (1965). Biosynthesis of cell-wall polysaccharide in mutant strains of Salmonella. III. Transfer of L-rhamnose and D-galactose. Biochemistry 4, 1550-1561.

NiKaIDo, H. \& NiKAIDO, K. (1965). Biosynthesis of cell-wall polysaccharide in mutant strains of Salmonella. IV. Synthesis of S-specific side-chains. Biochemical and Biophysical Research Communications 19, 322-327.

Nurminen, M., HellerQvist, C. G., Valtonen, V. V. \& MÄKELÄ, P. H. (1971). The smooth lipopolysaccharide character of 1,4,5,12 transductants formed as hybrids between groups $B$ and $\mathrm{D}$ of Salmonella. European Journal of Biochemistry 22, 500-505.

OSBORN, M. J. (1969). Structure and biosynthesis of the bacterial cell-wall. Annual Review of Biochemistry 38, 501-538.

Sawardeker, J. S., Slonecker, J. H. \& Jeanes, A. (1965). Quantitative determination of monosaccharides as their alditol acetates by gas liquid chromatography. Analytical Chemistry 37, 16021604.

SpICER, C. C. \& DATTA, N. (1959). Reversion of transduced antigenic characters in Salmonella 
typhimurium. Journal of General Microbiology 20, 136-143.

Staub, A. M. \& Bagdian, G. (1966). Etudes immunochimiques sur les Salmonella. XII. Analyse immunologique des facteurs 27A, 27B et 27D. Annales de l'Institut Pasteur 110, 849-860.

Stocker, B. A. D., Staub, A. M., Tinelli, R. \& KopaCKA, B. (1960). Etude immunochimique sur les Salmonella. VI. Étude de l'antigène 1 présent sur deux Salmonella des groupes B et E4. Annales de l'Institut Pasteur 98, 505-523.

Tinelli, R. \& Staub, A. M. (1960). Analyse de l'antigène $\mathrm{O}_{12}$ du tableau de Kauffmann-White.
Bulletin de la Société de chimie biologique 42, 583599.

WestPhal, O., Lüderitz, O. \& Bister, F. (1952). Über die Extraktion von Bakterien mit Phenol Wasser. Zeitschrift für Naturforschung 7b, 148155.

WhistleR, R. L. \& WolfRom, M. L. (1962). Methods in Carbohydrate Chemistry. I. Analysis and Preparation of Sugars. New York and London: Academic Press.

ZINDER, N. D. \& LederberG, J. (1952). Genetic exchange in Salmonella. Journal of Bacteriology 64, 679-699. 